(REVIEW ARTICLE)

\title{
Microcellulose Vs Nanocellulose - A Review
}

\author{
Michael Jacob Ioelovich * \\ Designer Energy, Chemical Department, Rehovot, Israel.
}

World Journal of Advanced Engineering Technology and Sciences, 2022, 05(02), 001-015

Publication history: Received on 18 January 2022; revised on 27 February 2022; accepted on 01 March 2022

Article DOI: https://doi.org/10.30574/wjaets.2022.5.2.0037

\begin{abstract}
Cellulose is the most abundant and renewable natural semi-crystalline polysaccharide. This biopolymer is an inexhaustible source of natural fibers (NFs), and valuable raw material for the production of microparticles of microcrystalline (MCC) and powdered cellulose (PC), as well as other cellulose micro-products, which are widely used in biomedicine, production of food additives, plastics, and other materials. In addition, cellulose has a nano-fibrillar architecture that promotes the release of free cellulose nanofibers (CNFs) and nanocrystals (CNCs). This review article describes the preparation methods, structural characteristics, properties, and applications of different types of micro(NFs, MCC, and PC) and nano-cellulose (CNFs, CNCs). Two main shortcomings hindering the wide application of various types of Nano cellulose (NC) were discovered, such as high production expenses and the difficulty of competing with commercial types of micro-cellulose. To reduce the production cost of $\mathrm{NC}$, a waste-free technology can be used, which allows completely utilize materials and chemicals, and produce cheap nanocrystalline aggregates (NCA) with zero emission of liquid and solid waste. Due to the low cost, such a nanostructured product, NCA, will be quite competitive with commercial micro-celluloses (MCC, PC, etc.) and can be used, e.g., as filler and thickener.
\end{abstract}

Keywords: Microcrystalline cellulose; Powdered cellulose; Nanofibers; Nanocrystals; Comparison; Critical analysis

\section{Introduction}

Cellulose is a natural, renewable, semi-crystalline polysaccharide that is the most abundant organic substance on Earth [1]. The main cellulose sources are plants [1-3]. The content of cellulose in herbaceous plants is $30-40 \%$, in woods 45 $50 \%$, in bast plants (flax, ramie, etc.) $60-70 \%$, and in cotton fibers upwards of $90 \%$ [4]. The main commercial sources of cellulose are wood and to a lesser extent - cotton. To isolate the cellulose, the plant feedstock is boiled in boilers under pressure in the presence of caustic soda (Soda process), a mixture of sodium hydroxide and sodium sulfide (Kraft process), or sulfurous acid and salts thereof (Sulfite process) [5]. Organosolv, oxidative, and some other pulping methods are also used, but on a lower scale. As a result, lignin, and hemicelluloses are removed, while cellulose is separated, washed, and dried. The production volume of wood pulp in the world is huge and reaches 250-300 million tons per year. Cotton cellulose is produced in smaller amounts, 20-25 million tons per year.

Cellulose is an ancient biopolymer known to mankind. For millennia, cellulose has served the needs of humankind in the form of ropes, nets, and fabrics made from cotton, flax, ramie, hemp, and other cellulose fibers. Historians have found that the earliest evidence for use of cellulose cotton fibers, dating back to 5,500 BC, was found in Baluchistan [6]. Various cellulose materials were used as far back as 5,000 years ago also in ancient Egypt. Cellulosic composites su ch as papyrus, reed, herbs, wood, etc. have also been known since ancient times.

\footnotetext{
${ }^{*}$ Corresponding author: Michael Jacob Ioelovich

Designer Energy, Chemical Department, Rehovot, Israel.. 
In the $2^{\text {nd }}$ century A.D. in China, a paper was invented, the production of which quickly spread to other countries. In the 8th century A.D., the paper started to be made in Samarkand. In the 13th century, the knowledge of paper technology spread from the Middle East to medieval Europe, where the first water-powered paper mills were built [7].

Cellulose was defined as a chemical substance related to polysaccharides in 1838 thanks to the works of French chemist Anselme Payen, who isolated it from plant matter and determined its chemical formula [8]. In the late 19th century, artificial cellulose fibers were developed as substitutes for natural silk [2]. At the beginning of the 20th century, Hermann Staudinger created the doctrine of synthetic and natural polymers. In particular, he proved that cellulose is a natural polymer consisting of thousands of monomeric units [9]. After the discovery of the synthesis of nitrocellulose and pyroxylin, the practical value of cellulose increased sharply. Cellulose research intensified, especially after the world wars. After the Second World War, the isolation methods, structure, properties, and various applications of cellulose were studied.

In the 70s of the $20^{\text {th }}$ century, Battista et al. proposed a new cellulose product called microcrystalline cellulose (MCC), which is obtained by acid hydrolysis of the initial cellulose to the minimum level-off degree of polymerization (LODP) corresponding to DP of individual crystallites [10]. Studies have shown that the MCC consists of aggregates of cellulose crystallites. The MCC has unique properties that make this microcrystalline powder indispensable as an excipient for tablets. Other applications of MCC also have been found, such as filler, dietary supplement, thickener, etc. As a result, a special industrial branch was created to produce MCC. Along with MCC, the production of powdered cellulose (PC) by dry grinding of initial cellulose materials was developed [11].

At the turn of the $20^{\text {th }}$ and $21^{\text {st }}$ centuries, nanoscience and nanotechnology came to the forefront of advanced knowledge, especially in nanomaterials, microelectronics, and microbiology. As a result, the nanoscience and nanotechnology were separated into a special knowledge area because objects of such Nano-sizes differ from micro-objects in such specific properties as a high specific surface area and surface adsorption capacity; increased Para crystallinity; small melting points, and increased solubility of Nano crystallites; the ability to aggregation, self-organization, and the formation of liquid-crystalline phases; high reactivity; increased transparency; etc.

Due to the immense popularity of nanotechnology and nanomaterials, cellulose chemists recalled that some researchers have already obtained nano-sized celluloses, although they called it differently, micelles [12], whiskers [13], nano-and microfibrils $[14,15]$. Cellulose nanotechnology is a part of general nanotechnology, operating on 1D, 2D, and 3D objects ranging in size from 1 to $100 \mathrm{~nm}$. The author of this review article together with co-authors, in their publications of the early 21st century, was probably one of the first, who propose calling cellulose micelles or whiskers nanoparticles [16, 17]. Since then, the number of publications on Nano cellulose (NC) has increased exponentially, from 100-150 to 3,0003,300 in 2019 [18]. A prediction shows that the number of publications on NC could reach 5,500-6,000 in 2022.

It was agreed to call crystalline nanoparticles of cellulose by nanocrystals (CNCs) $[19,20]$, and nano-fibrillated cellulose by nanofibers (CNFs) [20, 21]. In addition, bacterial nano-cellulose (BNC) [22], amorphous nano-cellulose (ANC) [23], and cellulose nano-yarn (CNY) [24] also were considered. Despite 20-30 years of research and the huge number of publications, industrial technology, and industrial production at least one type of Nano cellulose has not been created, which requires an explanation.

The main purpose of this review article was to describe the preparation methods, structural characteristics, properties, and uses of various types of micro-and nano-celluloses, as well as to discuss the advantages and disadvantages of these cellulose products.

\section{Microcellulose}

\subsection{Natural Cellulose Fibers}

Natural cellulose fibers of various origins have different sizes and shapes [1,4]. For example, fibers of cotton, flax, and rami have a length of several centimeters, while fibers of wood cellulose are short, 1-3 mm. In addition, natural cellulose fibers have a micro-size width, 10-30 $\mu \mathrm{m}$, and their wall thickness is 3-6 $\mu \mathrm{m}$. Cellulose fibers are applied in the papermaking, textile industry, and medicine $[25,26]$. In addition, they serve as reinforcers in various plastics that can be used in diverse applications such as furniture, sport and leisure goods, automotive, electronic appliances, building construction, boat industry, aerospace, etc. [26]. After delignification and bleaching, the cellulose fibers can be used as feedstocks in the production of cellulose derivatives, microcrystalline and powdered cellulose. 


\subsection{Microcrystalline cellulose}

Microcrystalline cellulose is a product of cellulose hydrolysis with dilute mineral acids [10]. The industrial production of MCC includes hydrolysis of the initial cellulose mainly with boiling 1-3 M hydrochloric acid, washing of the hydrolyzed cellulose, and neutralization to $\mathrm{pH}$ 5-7, after which the slurry is diluted, homogenized, and finally spray-dried [27]. The initial cellulose material used in the MCC industry can be bleached Kraft pulp or purified fibers of cotton cellulose. The resulting MCC particles formed after spray-drying have a spherical or elliptical shape with an average size of 50 to 200 $\mu \mathrm{m}$ depending on the parameters of the drying process. To reduce the particle size below $50 \mu \mathrm{m}$, the spray-dried MCC is subjected to additional grinding and screening. By changing the type of initial cellulose, the conditions of hydrolysis, spray-drying, and additional grinding it is possible to adjust the size and shape of the MCC particles, degree of crystallinity, DP, bulk density, moisture content, flowability, compactibility, and other properties.

Due to the unique combination of such characteristics as low DP, small particles size, high crystallinity, low solubility, high chemical, and biological purity, MCC is an irreplaceable excipient for tablets, filler for cosmetics, and dietary supplements for food products (Table 1).

Table 1 MCC Specification of USP

\begin{tabular}{|l|l|}
\hline Characteristics & Specification \\
\hline Appearance & A fine, white, odorless, crystalline powder \\
\hline DP & $<350$ \\
\hline Crystallinity & $70-80$ \\
\hline Av. particle size & $50-200 \mu \mathrm{m}$ \\
\hline Bulk density & $0.3-0.4 \mathrm{~g} / \mathrm{cm}^{3}$ \\
\hline pH & $5-7$ \\
\hline Loss of drying & $<7 \%$ \\
\hline Solubility in water & $<0.25 \%$ \\
\hline Solubility in ether & $<0.05$ \\
\hline Ash & $<0.05 \%$ \\
\hline Heavy metals & $<10 \mathrm{ppm}$ \\
\hline Total aerobic microbial count & $<100$ per g \\
\hline Total moulds and yeasts count & $<20$ per g \\
\hline Staphylococcus au., Pseudomonas aer., E. Coli, Salmonella & Absent \\
\hline
\end{tabular}

An additional opportunity to change the properties of MCC is the introduction of various functional additives before spray drying $[28,29]$. For example, the addition of mannitol to MCC dispersion enhances the disintegration of tablets compared to traditional MCC grades. The introduction of $\mathrm{SiO}_{2}$ improves the smoothness of tablets. The supplement of dibasic calcium phosphate or calcium sulfate to MCC dispersion facilitates compactibility, flowability, and reduces moisture uptake of tablets. A special colloidal type of MCC is produced by spray drying of MCC dispersion containing the addition of sodium carboxymethyl cellulose. This MCC type can be used as a suspending agent, emulsifier, and thickener [30].

At present, the production volume of MCC is 100-150 thousand tons per year. The market size of MCC was $\$ 885$ million in 2018 and is projected to reach more than $\$ 1.2$ billion by 2023 . The 7\% growth of the MCC market is triggered by the increasing demand in MCC for the production of food, pharmaceuticals, cosmetics, and personal care products [31]. In particular, the consumption of MCC in the food market will grow due to the demand for frozen microwave-compatible, low fat, and healthy food products. This growth is driven by innovations in frozen food packaging, changing consumer perceptions of the nutritional benefits of frozen foods, developing advanced refrigeration technologies, and producing healthy frozen foods having a low content of salts and calories due to an increase in dietary cellulose supplements. 
Top key manufacturers of MCC in the world are DowDuPont-FMC (USA), JRS Pharma (Germany), Asahi Kasei (Japan), Huzhou City Linghu Xinwang Chemical Co.(China), Mingtai and Wei Ming Pharma (Taiwan), Accent Microcell (India), Blanver (Brazil), etc. In China and other Asian counties, the production cost of MCC is \$2-3 per kg, and the market price is \$4-5 per kg. In USA and Europe, the production cost of MCC is estimated at \$4-5 per kg, while the market price is \$67 per kg.

Recently, a new waste-free "green" technology for the production of MCC has appeared, which simultaneously reduces also the production cost $[32,33]$. According to the proposed technology, the hydrolysis of the initial cellulose is carried out with boiling 1-2 M sulfuric acid. Then, the acidic MCC is separated from the acid, washed, diluted with water, and after homogenization, subjected to spray drying. Alternatively, the separated acidic MCC is not washed but neutralized with calcium oxide or hydroxide to obtain the slurry of $\mathrm{MCC}$ and $\mathrm{CaSO}_{4}$, which is diluted, homogenized, and spray dried to produce microparticles of MCC and $\mathrm{CaSO}_{4}$ composite. The spent sulfuric acid is mixed with acidic washing water and then neutralized with calcium oxide or hydroxide. As a result, calcium sulfate is formed, which can serve as a white pigment and a mineral binder:

$$
\begin{gathered}
\mathrm{H}_{2} \mathrm{SO}_{4}+\mathrm{CaO} \rightarrow \mathrm{CaSO}_{4}+\mathrm{H}_{2} \mathrm{O} \\
\mathrm{H}_{2} \mathrm{SO}_{4}+\mathrm{Ca}(\mathrm{OH})_{2} \rightarrow \mathrm{CaSO}_{4}+2 \mathrm{H}_{2} \mathrm{O}
\end{gathered}
$$

Thus, along with pure MCC or MCC composite, the by-product, $\mathrm{CaSO}_{4}$, is produced, the sale of which highly reduces the production cost of MCC and its composite. In addition, collected washing water is purified and returned to the technological cycle. The proposed technology makes it possible to introduce also other additives into the MCC. For example, if the acid is neutralized with hydroxylapatite, then pure MCC or MCC composite can be produced, along with superphosphate as a by-product. Such "green" technology utilizes the feedstock and chemicals completely and allows the production of inexpensive MCC or its composite with zero discharge of solid or liquid waste to the environment.

\subsection{Powdered Cellulose}

Powdered cellulose (PC) is produced by dry grinding of initial cellule materials. Various grinding techniques can be used such as ball mills, centrifugal grinding machines, hammer and knife mills, etc. Powdered cellulose is a white or almost white, odorless, and tasteless micro-powder of various particle sizes. To increase homogeneity, the pulverized cellulose micro-powder is screened to obtain the desired particle size.

The technical grades of PC are widely applied as a filler for various composites. In addition, these PC grades are good conductors of heat and electricity and hence, they are used in the production of welding electrodes. Pure PC grades can be used in cosmetics to improve texture and moisture levels, as well as a gentle abrasive for mechanical peeling. The additive of pure PC to food products can improve texture, increase water binding and reduce calories.

The known industrial PC grades are "Arbocel” produced by JRS GmbH (Germany) for diverse applications such as pharmaceutics, papermaking, polymer composites, chemistry, filtration, animal feed, welding electrodes, etc. [34]. Pure "Arbocel" grades are white, odorless, and tasteless powders having a particle size from 55 to $320 \mu \mathrm{m}$ and bulk density from 0.20 to $0.41 \mathrm{~g} / \mathrm{cm}^{3}$.

In addition to JRS GmbH (Germany), the main manufacturers of PC in the world are Mikro-Technik GmbH \& Co (Germany), Jelu-Werk J. Heeler GmbH \& Co (Germany), CFF GmbH \& Co and Belgium), Interfiber Sp. (Poland), NB Entrepreneurs (India), Accent Microcell Pvt. (India), Juku Orc hem Ltd (India), Fine Cell AB (Sweden), etc. The growth of the PC market has been estimated at 4.5\% [35]. The powdered cellulose is cheaper than MCC. The production cost of $\mathrm{PC}$ is about $\$ 1.5-2$ per $\mathrm{kg}$, while the market price is $\$ 2.5-3$ per $\mathrm{kg}$.

\section{Nanocellulose}

Cellulose is a nanostructured biopolymer consisting of bound nanofibrils [4]. Therefore, it is sufficient to apply intensive mechanical or ultrasonic energy to separate inter-fibrillar bonds and isolate the nanofibrillar bundles, i.e., cellulose nanofibers (CNFs). As is known, CNFs are built from ordered nanocrystals and low-ordered nanoscale amorphous domains (ADs). The nanocrystals having three-dimensional order are strong and inaccessible constituents. As opposite, the low-ordered ADs are weak and accessible places of the nanofibrils. Thereby, cleavage of chemical glycosidic bonds during hydrolysis occurs mainly in amorphous domains of nanofibrils, which facilitates the release of cellulose nanocrystals (CNCs). 
Currently, nano-cellulose (NC) is subdivided into five types: CNFs, CNCs, BNC, ANC, and CNY [19-25]. However, ANC is an experimental laboratory product. BNC is more of a nanostructured material rather than individual Nano-fibers. The electro-spun filaments of CNY have a diameter significantly greater than $100 \mathrm{~nm}$; therefore, these filaments are microfibers rather than nanofibers. Thus, if the Nano cellulose is discussed, then in fact two main types of NC should be considered, namely, cellulose nanofibers (CNFs) and cellulose nanocrystals (CNCs).

\subsection{CNFs}

CNFs can be isolated by intensive mechanical processing of purified cellulose [24]. For this purpose, three main disintegration methods of cellulose materials in an aqueous medium are used, such as high-pressure homogenization, micro-fluidization, and wet grinding.

High-pressure homogenization is a widely used method for lab-and pilot-scale production of CNFs [36-39]. This technique involves forcing the diluted aqueous dispersion of cellulose (usually with a concentration of $1 \%$ or lower) through a narrow gap with a diameter of 10-20 $\mathrm{m}$ using high pressure, from 40 to $200 \mathrm{MPa}$. The disintegration of cellulose until the CNFs formation is achieved as a result of the action of high shear forces on initial cellulose fibers, causing them to split into thin nanofibers. The degree of cellulose fibrillation at a constant concentration depends on the applied pressure and the number of homogenization cycles (passes). The higher the pressure, the higher the efficiency of cellulose disintegration (splitting) per one pass. The separation of cellulose into CNFs typically requires 15 passes to achieve the optimum aspect ratio [38].

Unlike the homogenizer, which operates at constant pressure, the micro-fluidizer operates at a constant shear rate [24]. The diluted cellulose dispersion (usually with the concentration of 1\% or lower) is pumped under increased pressure through a Z-shaped chamber, where it reaches a high shear force. To isolate CNFs, 15 passes are required at least.

Intensive wet grinding is a well-known technology used in the pulp and paper industry for many years. The process of separation of cellulose into nanoscale fibrils is carried out in an aqueous medium passing the diluted cellulose slurry between static and rotating grindstones (revolving speed of 1000-2000 rpm), which generates high shearing stress [24, 40]. After grinding (milling) of the cellulose dispersion to a high grinding degree (80-90 according to Shopper-Riegler degree), the dispersion of nanofibers can be obtained. A grinder uses shear forces to delaminate the surface of cellulose fibers and separate the CNFs. The degree of fibrillation is dependent on the distance between the grindstones, their material and roughness, concentration of cellulose in dispersion, and the number of passes of dispersion through the grinder. The isolation of CNFs requires a large number of passes and, therefore, a long grinding time and high energy consumption. Another disadvantage of this technology is the rapid wear of the grindstones, which requires their frequent repair and replacement.

Various mechanical processing usually produces a heterogeneous mixture of CNFs of various diameters ranging from 10 to $100 \mathrm{~nm}$, and lengths of several microns [41,42]. To improve homogeneity, the disintegration process must be prolonged to split the thickest nanofibers. Besides, the aqueous suspension of CNFs can be centrifuged to separate the coarse fraction of nanofibers.

When preparing CNF, a problem arises caused by a significant increase in the viscosity of the dispersion during disintegration, if the initial concentration of cellulose exceeds $2 \%$. It was found that the intensive mechanical treatment of $2 \%$ cellulose dispersion leads to a high increase in the surface area of forming CNFs, which causes the transformation of the liquid dispersion into a thick gel [43]. This phenomenon can completely stop further cellulose disintegration or drastically increase energy consumption. To avoid this problem, low concentrated cellulose dispersions, $1 \%$ or less must be used for the preparation of CNFs.

Another acute problem, especially for the production process of CNFs in a high-pressure homogenizer or microfluidizer, is the frequent clogging of these devices with relatively long and entangled cellulose fibers [24]. To overcome this disadvantage, as well as to reduce the energy consumption, the initial cellulose feedstock is subjected to various pretreatments, mechanical, ultrasound, cryo-crushing, chemical, biological, or a combination thereof, before intensive mechanical disintegration [44-47]. The main aim of such pretreatments is to reduce the length of initial cellulose fibers and to weaken the inter-fibrillar contacts inside these fibers to facilitate splitting. For example, after pretreatment of cellulose with cellulolytic enzyme, more uniform CNFs with a diameter of 10-30 nm can be obtained [43, 45]. Moreover, this pretreatment reduces energy consumption.

The aspect ratio of CNFs is in the range 70-100, which leads to a small percolation threshold, 0.7-1.0 vol. \% [48]. Thus, even small additions of these nanofibers can form a continuous network in a liquid medium or polymer matrix. The 
crystallinity and size of crystallites of obtained CNFs depend on the cellulose source and conditions of isolation of the nanofibers. It was found that degree of crystallinity of CNFs decreases slightly with an increase in the number of passes through the grinder [49]. The enzymatic pretreatment of initial cellulose does not change the crystalline structure [45, 50]. An exception is a pretreatment with specific enzymes, endoglucanases, which selectively destroy the amorphous domains of cellulose thus reducing the degree of crystallinity [51]. However, the nanofibers produced after preliminary carboxymethylation of initial cellulose have lower crystallinity and crystallites size than CNFs obtained by conventional mechanical processing without pretreatment [50]. On the other hand, pretreatment of cellulose with concentrated sulfuric acid leads to an increase in crystallinity and lateral size of crystallites.

The degree of polymerization of cellulose decreases both after enzymatic and chemical pretreatment and after intensive disintegration. The external specific surface area $\left(\mathrm{S}_{\mathrm{sp}}\right)$ of thick CNFs with a diameter of $100 \mathrm{~nm}$ is about $30 \mathrm{~m} / \mathrm{g}$. However, with a decrease in the diameter of nanofibers to $10 \mathrm{~nm}$, their $S_{\text {sp }}$ increases to $250 \mathrm{~m}^{2} / \mathrm{g}[48,50]$.

Theoretically, the dry CNFs oriented in an axial direction can have the axial Young modulus of about 110 GPa and tensile strength of about $2 \mathrm{GPa}$ [52]. However, the mechanical properties of real nanofibers are much lower, which is probably caused by a deflection of orientation from the axial axis. Indeed, it has been found that the average axial modulus of the dry cellulose nanofibers is about 30-36 GPa, and their tensile strength is about 600-700 MPa [52, 53].

The mechanical properties of dry films formed from water dispersions of CNFs resemble the characteristics of films made from regenerated cellulose having the modulus of 7-10 GPa, the tensile strength of 170-230 MPa, break elongation of $5-7 \%$, and density of $1.2-1.4 \mathrm{~g} / \mathrm{cm}^{3}[54,55]$. Films cast from aqueous dispersions of CNFs can be optically transparent if the cellulose nanofibers are densely packed, and the interstices between the fibers are small enough to avoid light scattering [56]. The thin films formed from dilute dispersions of CNFs had a denser structure and were optically translucent; the transparency value of the films at a wavelength of $600 \mathrm{~nm}$ was about 70-72\%. After additional polishing, the transmittance of CNFs films was improved and reached 78-90\%.

Flowable aqueous dispersions of CNFs have unique rheological properties such as pseudo-plasticity and shear thinning behavior [57]. Due to the high aspect ratio of the uncharged or low charged CNFs, the water dispersions of these nanofibers turn into a stable gel at relatively low concentrations, 2-3\%. The 3\% gel of CNFs has dynamic storage modulus $\geq 100 \mathrm{~Pa}$ and viscosity $\geq 50 \mathrm{~Pa}$ x sec. Due to its expressed hydrophilicity and high specific surface area, the network of CNFs can retain more than $97 \%$ water.

Potential applications of CNFs include various areas such as papermaking, polymer composites, insulation materials, medicine, cosmetics, food products, and some others. The obvious application of aqueous dispersions of CNFs is their use in paper compositions. It was found that the addition of $2-10 \%$ CNFs to paper composition also improves the mechanical properties of produced paper [58]. The Nano-paper made from CNFs exhibited quite high mechanical properties, and namely, the Young modulus (E) was $13 \mathrm{GPa}$ and the tensile strength (TS) was 214 MPa [59]. The Nanopaper produced from CNFs pretreated by TEMPO-mediated oxidation had higher mechanical properties than the Nanopaper from non-pretreated CNFs [60].

CNFs were also used to modify the surface of paper sheets. It was found that a thin layer $\left(1-2 \mathrm{~g} / \mathrm{m}^{2}\right)$ of CNFs on the paper surface creates a dense barrier against air permeability [61]. Preliminary carboxylated and carboxymethylated CNFs showed better barriers against $\mathrm{O}_{2}$, and grease than unmodified CNFs [62]. High barrier properties against $\mathrm{O}_{2}$ permeability can be created by coating the surface of paper or paperboard with an aqueous solution of polyvinyl alcohol (PVA) containing a small addition of CNFs, followed by drying [63]. A study of printing properties of paper was revealed that coating of its surface with the composition containing CNFs and starch highly reduces the linting and dusting propensity of the printing paper [64].

Various processing methods can be applied to make polymer nanocomposites reinforced by CNFs, such as solution casting [65-67], partial dissolution [47], melt-compounding [47,68], etc. The aqueous dispersions of CNFs can be mixed with aqueous solutions of various water-soluble polymers such as starch, amylopectin, albumin, gelatin, polyvinyl alcohol, polyethylene glycol, polyvinyl pyrrolidone, polyacrylamide, polyacrylic acid, urea-formaldehyde resin, melamine-formaldehyde resin, hydroxyethylcellulose, hydroxypropylcellulose, carboxymethylcellulose, methylcellulose, etc. After mixing, casting, and drying the nanocomposites are formed. For example, the introduction of CNFs dispersion to an aqueous solution of starch or plasticized starch followed by mixing, casting, and drying, leads to obtaining biodegradable nanocomposites with improved mechanical properties $[69,70]$.

The nanocomposites can be based also on water-insoluble but water-miscible polymers, i.e., latexes. For example, CNFs dispersion was mixed with polylactide latex, and then a nanocomposite film was cast, dried, and hot-pressed [71]. The 
introduction of CNFs dispersion of hydrogel into water solutions or latexes of polymers causes a strong thickening effect, which is of great importance to improve the features of water-borne adhesives, paints, coating compositions, and other water-based materials. A potential application area of CNFs is their use in water-borne wood adhesives based on urea, melamine, and some other resins [72]. The studies have shown that the addition of 1-2\% of CNFs to various adhesives significantly increases the bonding strength of wood samples.

The waterborne polymers, especially the waterborne PU, can be applied in 3D printing. However, such aqueous PU has a low viscosity, which is a problem for printing. To overcome this shortcoming, it was proposed to thicken the aqueous polymer with a hydrogel of CNFs $[73,74]$. Thus, the interaction between CNFs and PU opens a novel way to tune the viscosity of waterborne polymer for direct 3D printing and enhance the properties of the produced articles.

Some oligomeric resins are liquids, which can be easily mixed with Nano-fillers, e.g., with CNFs. Among such liquid resins, curable urethane and epoxy oligomers are most widely used in various applications, for example, in the production of protective coatings, paints, adhesives, sealants, insulators, foams, parts of machines, and other materials for various purposes.

To prepare PU-based nanocomposites, an aqueous dispersion of CNFs should be centrifuged to remove water excess and then washed with acetone to obtain organo-gel [75]. Next, this gel was mixed with a polyol in various ratios, and the mixture was sonicated to achieve a uniform distribution of the nano-filler and dried to remove acetone. Finally, the polyol-CNFs mixtures were treated with a diisocyanate to obtain polyurethane samples reinforced with CNFs. The study showed that due to the introduction of $1 \%$ CNFs to PU, the mechanical characteristics of the nanocomposite increased 2-4 times. The reinforcing effect of $1 \%$ CNFs is explained by the high aspect ratio of the nano-filler, which leads to a low percolation threshold, as well as by the chemical interaction between the hydroxyl groups of cellulose and diisocyanate.

Other known liquid oligomers are epoxy resins that are widely used for gluing, sealing, protective coating, production of textiles, and other composites. However, the cured epoxides are brittle and characterized by a low impact strength and poor crack growth resistance, which limits their applications. The incorporation of cellulose nanofibers into the epoxy resins can improve the properties of resulting nanocomposites. To prepare such nanocomposites CNFs hydrogel was washed and dispersed in acetone; then the CNFs dispersion in acetone was added dropwise to epoxy resin/hardener system, while intensive stirring; then cast films were cured [76]. The results have shown that the incorporation of a small addition of CNFs in the epoxy matrix significantly improved the thermal and mechanical characteristics of the composites.

To remove water from aqueous dispersions of CNFs before mixing with polymer resin, the freeze-drying method can be used [77]. As a result, porous aerogel was formed. Studies have shown that after freeze-drying lateral aggregation of CNFs occurs; therefore, the final aerogel contains microfibers with a diameter of 0.5 to $1.5 \mu$ m [74]. This aerogel was then impregnated with a liquid mixture of epoxy resin and hardener and cured into a solid composite. However, it was not nano-, but a micro-composite, since it contained microfibers instead of CNFs.

Thus, various drying methods, such as drying at elevated temperatures and freeze-drying, cannot be used to preserve the nano-size of CNFs. To overcome this obstacle, an indirect method of CNFs dehydration can be used, such as replacing water with a water-soluble organic solvent (acetone, alcohol, etc.), which is then replaced with a solvent that dissolves the chosen resin or polymer. Next, the organo-gel of CNFs is mixed with a solution of resin or polymer, after which the mixture is homogenized, cast, and evaporated until obtaining a solid nanocomposite. However, achieving a uniform distribution of dehydrated CNFs in a polymer melt is a difficult task.

In many cases, when preparing composites of polar CNFs with non-polar or low-polar resins or polymers, the problem of poor compatibility arises. There are several methods to improve compatibility between reinforcing filler and polymer matrix [44, 77-80], as follows:

- Chemical modification of CNFs-surface, e.g., by acetylation, silylation, etc.

- Grafting of CNFs-surface with short chains of hydrophobic polymers.

- Introduction of coupling agents-compatibilizers.

Freeze drying of aqueous dispersions of CNFs and some other methods are used to obtain highly porous and lightweight aerogels, which can be used, e.g., as insulating materials [81]. However, as it was shown earlier, as a result of freezedrying, the nanofibers are aggregated and therefore the resulting aerogels consist of microfibers rather than nanofibers. 
An interesting application of CNFs is their use as solid emulsifiers to stabilize 0/W emulsions [82]. Stabilization of the emulsions with characteristic micron-sized droplets was achieved at CNFs concentrations as low as $0.1 \%$.

Biomedical applications of CNFs are also being considered in such areas as excipients, bio-scaffolds, drug delivery vehicles, skin tissue repair, wound healing, etc. [83].

\subsection{CNCs}

Cellulose nanocrystals (CNCs) are usually isolated from cellulose materials by hydrolysis with 7-10 M mineral acids (5564 wt.\% sulfuric or 25-32 wt. \% hydrochloric acid) at moderate temperatures, $40-60^{\circ} \mathrm{C}$ until level-off DP (LODP), followed by mechanical or ultrasound disintegration in aqueous medium [4, 24, 46, 50]. Compared to sulfuric acid (SA), cellulose hydrolysis with hydrochloric acid $(\mathrm{HCl})$ requires higher temperatures to produce the CNCs of similar dimensions [84]. Further studies have shown that CNCs can be obtained also at lower concentrations of SA, 5-6 M, but at higher temperatures, $60-80^{\circ} \mathrm{C}[85]$.

The yield of isolated CNCs depends on the acid concentration. After cellulose hydrolysis with 64 wt. \% SA the yield of CNCs is $\leq 20 \%$ and with $63.5 \mathrm{wt}$. \% SA it is about 30\% [50,86]. When the concentration of SA is reduced to $40-60 \mathrm{wt}$. $\%$, the yield of CNCs increases to $50-70 \%[50,85]$.

The studies have shown that CNCs isolated from celluloses of various terrestrial plants have a lateral size in the range from 5 to $20 \mathrm{~nm}$, and length between 100 and $400 \mathrm{~nm}$; whereas the nanocrystals isolated from other sources, such as tunicate, algae, and bacterial cellulose, are longer and thicker [50, 80]. Moreover, the minimum dimensions of CNCs were close to the dimensions of individual nanocrystallites. However, the average and maximum dimensions of CNCs were higher than of individual nanocrystallites; this evidences the presence of nanocrystalline aggregates in isolated CNCs [50].

The isolated CNCs contain rod-like particles with an aspect ratio from 20 to 50. Due to this aspect ratio, the percolation threshold of CNCs is between 1.4 and 3.5 vol. \% [48]. Thus, the concentration of CNCs in a liquid medium or polymer matrix must be at least two times higher than that of CNFs to form a continuous network. The LODP of these nanoparticles can vary from 100 to 400 depending on the cellulose origin. The crystallinity degree of CNCs is high, 75$80 \%$. However, if the acid concentration was above $9 \mathrm{M}$, the crystallinity of obtained CNCs is reduced.

The mechanism of CNCs generation is a subject of discussion. This phenomenon is explained usually by selective acid hydrolysis of disordered amorphous domains of cellulose nanofibrils, whereas the more resistant nanocrystallites remained intact and can be isolated in a form of rod-like particles [87, 88]. However, this mechanism does not explain, why the CNCs cannot be isolated after cellulose hydrolysis with dilute acids, 1-3 M, despite achieving the LODP of cellulose and using mechanical or ultrasound disintegration [50, 89]. As is known, the hydrolysis of cellulose with dilute mineral acids up to LODP is used to produce microcrystalline cellulose (MCC) and not CNCs [10,17, 90]. The reason why it is necessary to use acids with increased concentrations to obtain CNCs is in the existence of strong lateral intercrystalline contacts between nanocrystallites of neighboring cellulose nanofibrils. These inter-crystalline contacts are inaccessible for dilute acids and remain intact after cellulose hydrolysis $[4,17,89]$. Therefore, to separate CNCs, it is not enough to hydrolyze only amorphous domains of cellulose, but it is necessary to corrode also inter-crystalline contacts, which is possible only under the action of mineral acids with a sufficiently high concentration, above $5 \mathrm{M}$ [85, 91]. As a result, the strong inter-crystalline contacts are replaced by weaker amorphous contacts. After breaking these contacts upon mechanical or ultrasound disintegration of hydrolyzed cellulose in the aqueous medium, the CNCs can be isolated and distributed in the dispersion.

The formation of negatively charged sulfonic groups on the CNCs surface after cellulose hydrolysis with sufficient concentrated sulfuric acid promotes electrostatic repulsion and leads to the phase stability of CNCs in aqueous dispersions [50, 84, 92]. Vice versa, the CNCs prepared by the hydrolysis of cellulose with hydrochloric acid are uncharged and therefore have a tendency to flocculate in an aqueous medium [84, 93].

The concentrated dispersions of charged CNCs show a pronounced birefringence [92, 94]. The investigation reveals that the dilute dispersion is isotropic since it contains randomly oriented nanoparticles. When a critical concentration is reached, the isotopic dispersion of CNCs turns into an anisotropic system having usually a chiral nematic ordered phase. The thin films cast from CNCs are transparent.

The mechanical properties of CNCs have been investigated by various methods. The calculation methods were based on the theory of micro-mechanics and the energies of chemical and hydrogen bonds [53, 95-98]. The experimental methods 
for the evaluation of the mechanical properties of CNCs included WAXS, Raman spectroscopy, and AFM [53, 97-100]. As follows from the calculations and experiments, the most probable value of axial Young modulus of CNCs is 140-160 GPa. The transversal modulus of CNCs is considerably lower, 15-30 GPa. This fact is explained by about a tenfold distinction between the total energies of bonds acting in axial and transverse directions of cellulose crystals [95]. The volume modulus has the lowest value, 5-6 GPa. The tensile strength of CNCs in the axial direction was about 8-10 GPa, while in the transversal direction about $1 \mathrm{GPa}$ [53].

When water-soluble polymers or polymer latexes are used, there are no problems with the miscibility of their water solutions or latexes with aqueous dispersions of CNCs. The polar CNCs also are compatible with various polar watersoluble polymers, such as starch, albumin, gelatin, polyvinyl alcohol, polyacrylic acid, polyethylene glycol, polyvinyl pyrrolidine, carboxymethylcellulose, etc. However, when water-insoluble non-or low-polar polymers are used to obtain nanocomposites, then aqueous dispersions of CNCs must be dehydrated by various methods. The studies showed that the conventional drying of aqueous dispersions of CNSs leads to aggregation of nanocrystals with forming of microparticles [50]. The freeze-drying also cannot prevent the aggregation process of CNCs [101, 102]. Even after ultrasonic treatment of the freeze-dried sample containing CNCs aggregates in non-polar (toluene, chloroform, etc.) or low-polar (acetone, alcohols, etc.) organic solvents, it was not possible to redisperse the CNCs [102]. The nanocrystalline aggregates of the freeze-dried CNCs can be redispersed after sonication only in water and highly polar organic solvents such as DMSO, DMFA, etc. [103].

The alternative is to use an indirect method of CNCs dehydration, such as replacing water with a water-soluble organic solvent, which is then replaced with a solvent that dissolves the chosen polymer. To prepare a solid nanocomposite, the formed organo-gel of CNCs is mixed with a polymer solution, and after homogenization, the mixture is cast and evaporated.

The CNCs can be chemically modified using all reactions known in cellulose chemistry such as etherification, esterification, cat ionization, oxidation, grafting, etc. [3, 46, 80, 94, 104, 105]. The surface-modified CNCs containing hydrophobic groups exhibit improved compatibility with non-or low-polar polymers in various polymers composites. In addition, such modified CNCs form stable colloids in organic solvents [105].

Apart from the use of CNCs as fillers for various polymers, other potential applications of unmodified and modified CNCs are also considered, such as nano-carriers of catalysts, strengthening additives to paper and its coating compositions, barrier additives for food packaging, thickeners of aqueous systems, solid nano-emulsifiers of Pickering emulsions, nano-excipients and nano-carriers of medicaments, functional nano-agents for biomedicine and cosmetics, etc. [106, 107].

\section{Discussion}

Despite many publications on the promising potential for the application of various types of nano-cellulose (NC), this potential has not been implemented. This is caused by the fact that NC does not have a unique scope. Currently, practically all potential applications of NC are occupied by available and cheap common cellulose products. For example, in the production of polymer composites, micro-sized natural cellulose fibers, powdered cellulose, wood flour, and some other cellulose-based commercial micro-fillers are used [34, 108-111]. Various natural fibers also are used in the packaging compositions [112]. These composites have low cost, meet standards, satisfy consumers, and, therefore, do not require the replacement of common cellulose fibers and fillers with NC.

Diverse paper types have standard compositions and properties that do not need in NC additives. When improvement of paper properties is required, inexpensive commercial substances are used for this purpose. Natural cellulose fibers are the main feedstock used for the production of cheap insulation materials [113]. In addition, aerogels can be obtained by a simple technology using cheap commercial cellulose materials instead of NC [114]. To thicken and stabilize dispersions and emulsions, colloidal types of microcrystalline cellulose $[30,115]$, as well as a huge number of cheap synthetic thickeners and emulsifiers, are used.

The practical use of NC in biomedicine cannot currently be implemented, since, unlike MCC, neither the CNFs nor the CNCs have permission from the US FDA and regulatory medical institutions of other counties.

Another reason why NC cannot compete with conventional commercial cellulose materials (fibers, MCC, PC, etc.) is the high production cost both of CNFs and CNCs. So, Japanese researchers estimate the minimum cost of CNFs at $\$ 44$ per kg DM [116]. In a 2017 study, the cost of TEMPO-oxidized CNFs was estimated at $\$ 17$ per kg DM, taking into account only energy costs and minimal consumption of chemicals [117]. However, since 2017, the cost of energy and chemicals has 
increased 2.3-2.5 times, and currently, the cost of CNFs is \$30-35 per kg DM. And that is not the full production cost yet as it did not include salary, equipment depreciation, overhead costs, etc.

Such a high cost of CNFs production is explained by the low concentration ( $\mathrm{C} \leq 1 \%$ ) of cellulose used for disintegration in the aqueous medium to isolate nanofibers without gel-forming. The lower the pulp concentration, the higher the energy cost per unit of dry mass (for example, per $1 \mathrm{~kg} \mathrm{DM}$ ) of the final nano-product, and the higher the production cost of CNFs. Pretreatment allows to reduce the energy consumption for disintegration and total production cost of CNFs, but not more than $50 \%$, since simultaneously the cost for pretreatment increases.

The production cost of CNCs is also quite high due to the high acid consumption at the hydrolysis stage and the increased energy consumption required for hydrolysis, the disintegration of nanocrystalline aggregates, concentration by evaporation, freeze-drying, etc. It was calculated that the cost of hydrolysis and evaporation steps is estimated at \$2030 per kg DM [118]. If take into account the full expenses, including the disintegration, salary, equipment depreciation, overhead costs, and others, then the actual production cost of CNCs can reach \$30-40 per kg DM.

Currently, in some countries, there are pilot plants with a capacity of 70 to $300 \mathrm{~kg}$ per hour, where the technology is being developed and samples of CN are prepared to study their structure and properties [119]. For example, a Canadian company “Cellulose Lab" sells small NC samples at a huge price of $\$ 2,000-8,000$ per kg DM [120]. The price of pretreated NC samples is even higher.

The very high production cost of CNFs and CNCs is a significant obstacle to the widespread use of NC. Calculations have shown that along with high acid consumption, the stages of disintegration of hydrolyzed cellulose and evaporation of dilute aqueous dispersions of CNCs are very expensive and significantly increase the total production cost of nanocrystalline cellulose. Therefore, it is most advantageous to eliminate these stages and utilize the used sulfuric acid.

For this purpose, the waste-free technology of CNCs was proposed [118]. According to this technology, the hydrolyzed cellulose, containing nanocrystalline aggregates (NCA), is separated from sulfuric acid, and after washing is concentrated into $40 \%$ paste without disintegration and evaporation. Further, the separated sulfuric acid is neutralized with a hydroxyapatite or calcium base to obtain a valuable by-product, sale of which significantly reduces the cost of the primary product, NCA. Wastewater was purified and returns to the technological cycle. Thus, this waste-free technology allows the complete utilization of materials and chemicals and produces cheap NCA with zero emission of liquid and solid waste.

The production cost of NCA is estimated at \$3.5-4.0 per kg DM, i.e., becomes comparable to the production cost of commercial MCC. The obtained semi-finished product, NCA, or its composite with the white pigment $\left(\mathrm{CaSO}_{4}\right)$ are intended for sale to consumers, who can mix them with other components and then grind if there is a need to destroy aggregates and isolate free nanoparticles.

\section{Conclusion}

Numerous studies on the isolation and characterization of NC are of great scientific importance for elucidating the supramolecular structure of cellulose of various origins. However, the practical use of NC encounters significant difficulties associated with its high production cost and the fact that potential areas of NC application are already occupied by commercial cellulose micro-sized materials such as cellulose fibers, MCC, PC, etc. As a result, the profitable industrial production of NC was not implemented. Currently, there are only pilot plants, where the technology is being developed and samples of NC are obtained. Due to the very high cost of NC, the prospects for their practical use are doubtful. However, if instead of free CNCs, a semi-finished product containing nanocrystalline aggregates (NCA) is produced using waste-free technology, then such a nanostructured product, NCA, will be quite competitive with commercial MCC and can be used, e.g., as filler and thickener.

\section{Compliance with ethical standards}

\section{Disclosure of conflict of interest}

The author of this paper hereby declares that there is no conflict of interest. 


\section{References}

[1] Seddiqi H, Oliaei E, Honarkar H, et al. Cellulose and its derivatives: towards biomedical applications. Cellulose. 2021; 28: 1893-1931.

[2] Lavanya D, Kulkarni PK, Dixit M, et al. Sources of cellulose and their applications - a review. IJDFR. 2011; 2: 19 38.

[3] Klemm D, Heublein B, Fink H-P, Bohn A. Cellulose: fascinating biopolymer and sustainable raw material. Angew. Chem. 2005; 44: 2-37.

[4] Ioelovich M. Cellulose: Nanostructured Natural Polymer. Lambert Academic Publishing, Saarbrücken. 2014; 88.

[5] Sjöström E. Wood Chemistry, Fundamentals, and Applications. Academic Press, London. 1993; 290.

[6] Moulherat C, Tengberg M, Haquet JR, Mille BT. First Evidence of Cotton at Neolithic Mehrgarh, Pakistan. J. Archaeologic. Sci. 2002; 29(12): 1393-1401.

[7] Murray S. The Library: An Illustrated History. Skyhorse Publishing, New York. 2009; 328.

[8] Payen A. Mémoire sur la composition du tissupropre des planteset du ligneux. Comptesrendus. 1838; 7: 1052 1056.

[9] Percec V, Xiao Q. From organic chemistry to chemical biology via macromolecules with Hermann Staudinger. Giant. 2020; 4: 1-9.

[10] Battista OA, Smith PA. Microcrystalline cellulose. Ind. Eng. Chem. 1962; 54(9): 20-29.

[11] Korpela A, Orelma H. Manufacture of fine cellulose powder from chemically crosslinked Kraft pulp sheets using dry milling. Powder Technol. 2020; 361: 642-650.

[12] Ränby BG. The cellulose micelles. TAPPI. 1952; 35: 53-58.

[13] Favier V, Chanzy H, Cavaille JY. Polymer nanocomposites reinforced by cellulose whiskers. Macromolecules. 1995; 28(18): 6365-6367.

[14] Herrick FW, Casebier RL, Hamilton J.K., Sandberg K.R. Microfibrillated cellulose: morphology and accessibility. J. Appl.Polym. Sci. Appl. Polym. Symp. 1983; 37: 797-813.

[15] Turbak AF, Snyder FW, Sandberg KR. Microfibrillated cellulose, f new cellulose product, properties, uses and commercial potential. J. Appl. Polym. Sci. Appl. Polym. Symp. 1983; 37: 815-827.

[16] Ioelovich M, Leykin A. Nano-cellulose and its applications. SITA. 2004; 6(3): 17-24.

[17] Ioelovich M. Formation nano-structure of microcrystalline cellulose. Cell. Chem. Technol. 2006; 40(5): 313-317.

[18] Dias OAT, Konar S, Leão AL et al. Current state of applications of nanocellulose in flexible energy and electronic devices. Frontiers in Chem. 2020; 8(420): 1-16.

[19] Xie Sh, Zhang X, Walcott MP, Lin H. Applications of cellulose nanocrystals: a review. Eng. Sci. 2018; 2: 4-16.

[20] Hindi Sh SZ. Differentiation and synonyms standardization of amorphous and crystalline cellulosic products. Nanosci. Nanotech. Res. 2017; 4: 73-85.

[21] Trache D, Tarchoun AF, Derradji M et al. Nanocellulose: from fundamentals to advanced applications. Frontiers in Chemistry. 2020; 8(392): 1-33.

[22] Klemm D, Schumann D, Kramer F et al. Nanocellulose materials - different cellulose, different functionality. Macromol. Symp. 2009; 280: 60-71.

[23] Ioelovich M. Preparation, characterization and application of amorphized cellulose - a review. Polymers. 2021; 13: $1-21$.

[24] Kargarzadeh H, Ioelovich M, Ahmad I, Thomas S, Dufresne A. Methods for Extraction of Nanocellulose from Various Sources, in: Handbook of Nanocellulose and Cellulose Nanocomposites. Wiley, Singapore. 2017; 1-50.

[25] Ioelovich M. Nanocellulose - Fabrication, Structure, Properties and Application in the Area of Care and Cure, in: Fabrication and Self-Assembly of Nanobiomaterials: Applications of Nanobiomaterials. Edited by Alexandru Mihai Grumezescu. Elsevier, Amsterdam. 2016; 9: 243-288. 
[26] Karimah A, Ridho MR, Munawar SS et al. A comprehensive review on natural fibers: technological and socioeconomical aspects. Polymers. 2021; 13: 1-27.

[27] Landín M, Martínez-Pacheco R, Gómez-Amoza JL, et al. Effect of batch variation and source of pulp on the properties of microcrystalline cellulose. Int. J. Pharmaceut. 1993; 91: 133-141.

[28] Jivraj M, Martini LG, Thomson CM. An overview of the different excipients useful for the direct compression of tablets. Pharmaceut. Sci. Techn. Today. 2000; 3: 58-63.

[29] Hentzschel CM, Sakmann A, Leopold CS. Comparison of traditional and novel tableting excipients: Physical and compaction properties. Pharmaceut. Develop. Technol. 2012; 17: 649-653.

[30] VIVAPUR MCG Dispersible Cellulose. Brochure of JRS Pharma. 2018.

[31] Microcrystalline Cellulose (MCC) Market Size. Industry Analysis Report, Application Growth Potential, Price Trends, Competitive Market Share and Forecast, 2019 - 2026. Global Market Insight. 2018; 184.

[32] Ioelovich M. Green technology of microcrystalline cellulose. SITA. 2018; 20: 3-7.

[33] Ioelovich M. Green chemistry of micro-and nanoparticles of plant biomass. South Asian Res. J. Nat. Products. 2018: 1: 1-10.

[34] ARBOCEL Powdered Cellulose. Markan Global Enterprises Inc. 2022.

[35] Powdered Cellulose Global Market Forecast, Trend Analysis and Opportunity Assessment to 2030. Business Wire. 2021.

[36] Kalia S, Boufi S, Celli A, Kango S. Nanofibrillated cellulose: surface modification and potential applications. Colloid and Polymer Science. 2014; 292(1): 5-31.

[37] Ren S, Sun X, Lei T, Wu Q. The effect of chemical and high-pressure homogenization treatment conditions on the morphology of cellulose nanoparticles. J. Nanomaterials. 2014; 1-11.

[38] Lee SY, Chun SJ, Kang IA, Park JY. Preparation of cellulose nanofibers by high-pressure homogenizer and cellulose-based composite films. J. Indian Eng. Chem. 2009; 15(1): 50-55.

[39] Davoudpour Y, Hossain S, Khalil HP. Optimization of high-pressure homogenization parameters for the isolation of cellulosic nanofibers using response surface methodology. Industr. Crops and Products. 2015; 74: $381-387$.

[40] Missoum K, Belgacem MN, Bras J. Nanofibrillated cellulose surface modification: a review. Materials. 2013; 6(5): 1745-1766.

[41] Siqueira G, Bras J, Dufresne A. Cellulosic bionanocomposites: A review of preparation, properties and applications Polymers. 2010; 2: 728-765.

[42] Jonoobi M, Oladi R, Davoudpour Y et al. Different preparation methods and properties of nano-structural cellulose 1 from various natural resources and residues: A review. Cellulose. 2015; 22(2): 935-969.

[43] Ankerfors M. Microfibrillated cellulose: Energy-efficient preparation techniques and key properties. KTH Royal Institute of Technol, Stockholm. 2012; 49.

[44] Siro I, Plackett D. Microfibrillated cellulose and new nanocomposite materials: a review. Cellulose. 2010; 17: 459494.

[45] Henriksson M, Henriksson G, Berglund LA, Lindstròm T. An environmentally friendly method for enzymeassisted preparation of microfibrillated cellulose nanofibers. European Polym. J. 2007; 43(8): 3434-3441.

[46] Hubbe MA, Rojas OJ, Lucia LA, Sain M. Cellulosic nanocomposites - a review, Bioresources. 2008; 3(3): $929-980$.

[47] Eichhorn SJ, Dufresne A, Aranguren M et al. Review: current international research into cellulose nanofibres and nanocomposites. J. Mater. Sci. 2010; 45(1): 1-33.

[48] Ioelovich M. Cellulose nanofibers and their nanocomposites - A review. SITA. 2021; 23(1-2): 86-99.

[49] Iwamoto S, Nakagaito AN, Yano H. Nano-fibrillation of pulp fibers for the processing of transparent nanocomposites. Appl. Physics. 2007; 89(2): 461-466.

[50] Ioelovich M. Characterization of various kinds of nanocellulose, in: Handbook of Nanocellulose and Cellulose Nanocomposites. Wiley, Singapore. 2017; 51-100. 
[51] Chiriac AI, Pastor FI, Popa VI et al. Changes of supramolecular cellulose structure and accessibility induced by the processive endoglucanase Cel9B from Paenibacillus barcinonensis. Cellulose. 2014; 21(1): 203-219.

[52] Tanpichai S, Quero F, Nogi M et al. Effective Young's modulus of bacterial and microfibrillated cellulose fibrils in fibrous networks. Biomacromol. 2012; 13(5): 1340-1349.

[53] Ioelovich M. Structure and mechanical properties of cellulose nanocrystallites. SITA. 2012; 14(3): 91-99.

[54] Henriksson M. Cellulose Nanofibril Networks and Composites - Preparation, Structure and Properties. KTH: Stockholm. 2008.

[55] Fukuzumi H. Studies on Structure and Properties of TEMPO-oxidized Cellulose Nanofibril Films. Tokyo University: Tokyo. 2011.

[56] Dufresne A. Nanocellulose: a new ageless bionanomaterial. Mater. Today. 2013; 16(6): 220-227.

[57] Paakko M, Ankerfors M, Kosonen H et al. Enzymatic hydrolysis combined with mechanical shearing and highpressure homogenization for nanoscale cellulose fibrils and strong gels. Biomacromol. 2007; 8(6): 1934-1941.

[58] Boufi S, González I, Delgado-Aguilar M et al. Nanofibrillated cellulose as an additive in papermaking process: a review. Carbohyd.Polym. 2016; 154: 151-166.

[59] Henriksson M, Berglund LA, Isaksson P, et al. Cellulose nanopaper structures of high toughness. Biomacromolecules. 2008; 9(6): 1579-1585.

[60] Gindl-Altmutter W, Veigel S, Obersriebnig M et al. High-modulus oriented cellulose nanopaper, in: Functional Materials from Renewable Sources. ACS Symp. Series. ACS, Washington DC. 2012; 3-16.

[61] Syverud K, Stenius P. Strength and barrier properties of MFC films. Cellulose. 2009; 16: 75-85.

[62] Aulin C, Gällstedt M, Lindström T. Oxygen and oil barrier properties of micro fibrillated cellulose films and coatings, Cellulose. 2010; 17: 559-574.

[63] Wang J, Gardner DJ, Stark NM, et al. Moisture and oxygen barrier properties of cellulose nanomaterial-based films. Sustainable Chem. Eng. 2018; 6: 49-70.

[64] Song H, Ankerfors M, Hoc M, Lindstrom T. Reduction of the linting and dusting propensity of newspaper using starch and microfibrillated cellulose. Nord. Pulp Pap. Res. J. 2010; 25: 495-504..

[65] Babaee M, Jonoobi M, Hamzeh Y, Ashori A. Biodegradability and mechanical properties of reinforced starch nanocomposites using cellulose nanofibers. Carbohydrate Polym. 2015; 132: 1-8.

[66] Santos FA, Tavares MIB. Development of biopolymer/cellulose/silica nanostructured hybrid materials and their characterization by NMR relaxometry. Polymer Testing. 2015; 47: 92-100.

[67] Lee K-Y, Aitomäki Y, Berglund LA et al. On the use of nanocellulose as reinforcement in polymer matrix composites. Composites Sci. Technol. 2014; 105: 15-27.

[68] Dos Santos FA, Iulianelli GCV, Tavares MIB. The use of cellulose nanofillers in obtaining polymer nanocomposites: properties, processing, and applications. Mater. Sci. Appl. 2016; 7(5): 1-20.

[69] Ilyas RA, Sapuan SM, Ishak MR. et al. Nanocellulose reinforced starch polymer composites: a review of preparation, properties and application, in: Proceedings of 5th Int. Conf. on Applied Sci. Eng. Cameron Highlands. 2018; 325-341.

[70] Karimi S, Tahir P, Dufresne A, et al. A comparative study on characteristics of nanocellulose reinforced thermoplastic starch biofilms prepared with different techniques. Nordic Pulp \& Paper Res. J. 2014; 29(1): 4145.

[71] Larsson K, Berglund L, Ankerfors A, Lindstrom T. Polylactide latex/nanofibrillated cellulose bionanocomposites of high nanofibrillated cellulose content and nanopaper network structure prepared by a papermaking route. J. Appl. Polym. Sci. 2012; 125(3): 2460-2466.

[72] Vineeth SK, Gadhave RV, Gadekar PT. Nanocellulose applications in wood adhesives -review. Open J. Polym. Chem. 2019; 9: 63-75.

[73] Chen R-D, Huang C-F, Hsu S-H. Composites of waterborne polyurethane and cellulose nanofibers for 3D printing and bio-applications. Carbohydrate Polym. 2019; 212: 75-88. 
[74] Athukoralalage SS, Balu R, Dutta NK, Choudhury NR. 3D bioprinted nanocellulose-based hydrogels for tissue engineering applications: a brief review. Polymers. 2019; 11: 1-13.

[75] Ivdre A, Mucci V, Stefani PM, Aranguren MI, Cabulis U. Nanocellulose reinforced polyurethane obtained from hydroxylated soybean oil. IOP Conf. Ser. Mater. Sci. and Eng. 2016; 111(1): 1-5.

[76] Roszowska-Jarosz M, Masiewicz J, Kostrzewa M, et al. Mechanical properties of bio-composites based on epoxy resin and nanocellulose fibres. Materials. 2021; 14: 1-16.

[77] Nissilä T, Wei J, Geng Sh, et al. Ice-templated cellulose nanofiber filaments as a reinforcement material in epoxy composites. Nanomaterials. 2021; 11: 1-15.

[78] Khalil HPS, Bhat AH, Yusra I. Green composites from sustainable cellulose nanofibrils: a review. Carbohyd. Polym. 2012; 87: 963- 979.

[79] Missoum K, Belgacem MN, Bras J. Nanofibrillated cellulose surface modification: a review. Materials. 2013: 6(5): 1745-1766.

[80] Moon RJ, Martini A, Nairn J et al. Cellulose nanomaterials review: structure, properties, and nanocomposites. Chem. Soc. Rev. 2011; 40: 3941-3994.

[81] Chen Y, Zhang L, Yang Y et al. Recent progress on nanocellulose aerogels: preparation, modification, composite fabrication, applications. Adv. Mater. 2021; 33: 1-34.

[82] Bai L, Huan S, Xiang W, Rojas OJ. Pickering emulsions by combining cellulose nanofibrils and nanocrystals: phase behavior and depletion stabilization. Green Chem. 2018; 20: 1571-1582.

[83] Lin N, Dufresne A. Nanocellulose in biomedicine: current status and future prospect. European Polym. J. 2014; 59: 302-325.

[84] Li Y, Ragauskas A. Cellulose nano-whiskers as a reinforcing filler in polyurethanes. Adv. Diverse Industr. Appl. Nanocompos. 2011; 3: 17-36.

[85] Ioelovich M. Superposition of acid concentration and temperature at production of nanocrystalline cellulose particles. J. Chemistry: Education, Research and Practice. 2017; 1(1): 1-4.

[86] Bondeson D, Mathew A, Oksman K. Optimization of the isolation of nanocrystals from microcrystalline cellulose by acid hydrolysis. Cellulose. 2006; 13: 171-180.

[87] Said MA, Samir A, Alloin F, Paillet M, Dufresne A. Tangling effect in fibrillated cellulose reinforced nanocomposites. Macromolec. 2004; 37(11): 4313-4316.

[88] Xie H, Du H, Yang X, Si Ch. Recent strategies in preparation of cellulose nanocrystals and cellulose nanofibrils derived from raw cellulose materials. Int. J. Polym. Sci. 2018; 7923068: 1-25.

[89] Ioelovich M. Models of supramolecular structure and properties of cellulose. J. Polym. Sci. Ser. A. 2016; 58: 925943.

[90] Nada A, El-Kady M, Abd El-Sayed E, Amine F. Preparation and characterization of microcrystalline cellulose. Bioresources. 2009; 4(4): 1359-1371.

[91] Ioelovich M. Study of conversion of cellulose into micro-and nanocrystalline particles during acidic hydrolysis. Res. J. Nanosci. Eng. 2018; 2(4): 10-13.

[92] Lin N, Dufresne A. Surface chemistry, morphological analysis and properties of cellulose nanocrystals with gradiented sulfation degrees. Nanoscale. 2014; 6: 5384-5393.

[93] Araki J, Wada M, Kuga S, Okana T. Influence of surface charge on viscosity behavior of cellulose microcrystal suspension. J. Wood Sci. 1999; 45(3): 258-261.

[94] Habibi Y, Lucia LA, Rojas OJ. Cellulose nanocrystals chemistry, self-assembly and applications. Chem. Rev. 2010; 110(6): 3479-3500.

[95] Eichhorn SJ, Davies GR. Modeling the crystalline deformation of native and regenerated cellulose. Cellulose. 2006; 13(3): 291-307.

[96] Tanaka F, Iwata T. Estimation of the elastic modulus of cellulose crystal by molecular mechanics simulation. Cellulose. 2006; 13(5): 509-517.

[97] Wu X, Wagner R, Raman A, et al. Elastic deformation mechanics of cellulose nanocrystals. TMS. 2010; 2: 689-696. 
[98] Lahiji R, Xu X, Reifenberger R, et al. Atomic force microscopy characterization of cellulose nanocrystals. Langmuir. $2010 ; 26(6): 4480-4488$.

[99] Nishino T, Takano K, Nakamae K. Elastic modulus of the crystalline regions of cellulose polymorphs. J. Polym. Sci. Polym. Phys. 1995; 33(11): 1647-1651.

[100] Šturcová A, Davies GR, Eichhorn SJ. Elastic modulus and stress-transfer properties of Tunicate cellulose whiskers. Biomacromolecules. 2005; 6(2): 1055-1061.

[101] Han J, Zhou C, Wu Y, et al. Self-assembling behavior of cellulose nanoparticles during freeze-drying: effect of suspension concentration, particle size, crystal structure, and surface charge. Biomacromolecules. 2013; 14: 1529-1540.

[102] Voronova MI, Surov OV, Rubleva NV, et al. Dispersion of nanocrystalline cellulose in organic solvents. Chemistry of Raw Mater. 2019; 1: 39-50.

[103] Viet D, Beck-Candanedo S, Gray DG. Dispersion of cellulose nanocrystals in polar organic solvents. Cellulose. 2007; 14: 109 -113.

[104] Thakur V, Guleria A, Kumar S, et al. Recent advances in nanocellulose processing, functionalization and applications: a review. Mater. Adv. 2021; 2: 1872-1895.

[105] Peng Sh, Luo Q, Zhou G, Xu X. Recent advances on cellulose nanocrystals and their derivatives. Polymers. 2021; 13: 3247: 1-34.

[106] Xie Sh, Zhang X, Walcott MP, Lin H. Applications of cellulose nanocrystals: a review. Eng. Sci. 2018 ; 2: 4-16.

[107] Dhar N. Novel Cellulose Nanoparticles for Potential Cosmetic and Pharmaceutical Applications, University Press, Waterloo. 2010; 207.

[108] Girijappa YGT, Rangappa SM, Parameswaranpillai J, Siengchin S. Natural fibers as sustainable and renewable resource for development of eco-friendly composites: a comprehensive review. Frontiers in Mater. 2019; 6(226): $1-14$.

[109] Stanaszek-Tomal E. Wood - polymer composites as an alternative to the natural environment. Mater. Sci. Eng. 2019; 603(022009): 1-8.

[110] Chan CM, Vandi L-J, Pratt S, et al. Composites of wood and biodegradable thermoplastics: a review. Polymer Reviews. 2018; 58(3): 1-51.

[111] Paridah MT, Juliana AH, Zaidon A, et al. Nonwood-based composites. Curr. Forestry Rep. 2015; 1: 221-238.

[112] Johansson C, Bras J, Mondragon I, et al. Renewable fibers and bio-based materials for packaging applications - a review of recent developments. Bioresources. 2012; 7(2): 2506-2552.

[113] Hurtado PL, Rouilly A, Maréchal VV, Raynaud C. A review on the properties of cellulose fibre insulation. Building and Environment. 2016; 96: 170-177.

[114] Long L-Y, Weng Y-X, Wang Y-Z. Cellulose aerogels: synthesis, applications, and prospects. Polymers. 2018; 10(623): 1-28.

[115] Oza KP, Frank SG. Microcrystalline cellulose stabilized emulsions. J. Dispersion Sci. Technol. 1986; 7(5): 543-561.

[116] Yuzawa M. Move over carbon fiber, here comes cellulose nanofiber. Nikkei. 2017; 2: 1-3.

[117] Serra A, González I, Oliver-Ortega $\mathrm{H}$ et al. Reducing the amount of catalyst in TEMPO-oxidized cellulose nanofibers: effect on properties and cost. Polymers. 2017; 9(557): 1-14.

[118] Ioelovich M. Waste free technologies for production of nanocrystalline cellulose and its composites. Adv. Env. Waste Manag. Recycling. 2020; 3(2): 128-132.

[119] Topunov EA, Sevastyanova JV. Powder cellulose materials: a review, classification, characteristics and applications. Chem. Plant Mater. 2021; 4: 31-45.

[120] Cellulose Lab. Nanocellulose Products Price. nanocellulose price; cellulose lab. 2020. 